\title{
Medo e ansiedade de estudantes de Odontologia diante da pandemia do novo coronavírus: um estudo transversal
}

Fear and anxiety in dental students due to new coronavirus pandemic: a cross-sectional study Miedo e ansiedad en estudiantes de Odontología ante la nueva pandemia de coronavirus: un estudio transversal

\author{
Rafaela Zazyki de ALMEIDA ${ }^{1}$ \\ Maísa CASARIN ${ }^{2}$
}

Bruna Oliveira de FREITAS ${ }^{1}$

Francisco Wilker Mustafa Gomes MUNIZ

${ }^{1}$ Curso de graduação. Faculdade de Odontologia, Universidade Federal de Pelotas (UFPEL) 960105-560 Pelotas-RS, Brasil

${ }^{2}$ Docente, Departamento de Semiologia e Clínica, Programa de Pós-Graduação em Odontologia, Área de Periodontia, Faculdade de Odontologia da Universidade Federal de Pelotas (UFPEL) 960105-560 Pelotas-RS, Brasil

\section{Resumo}

Objetivo: Esse estudo objetivou investigar percepções de estudantes de Odontologia quanto ao medo e à ansiedade em relação ao manejo de pacientes e ao risco de infecção por COVID-19. Materiais e métodos: Esse estudo transversal envolveu todos os alunos regularmente matriculados em Odontologia, no primeiro semestre de 2020, da Universidade Federal de Pelotas. Um questionário foi aplicado, coletando dados demográficos, nível de formação e perguntas relacionadas ao medo e ansiedade frente à pandemia de COVID-19. Quatro comparações de acordo com a fase da graduação (fase pré-clínica ou clínica), nível de graduação e pós-graduação e de acordo com os sexos foram feitas. Análises independentes para as comparações entre os sexos foram realizadas para os alunos de graduação e de pós-graduação $(\alpha<5 \%)$. Resultados: Foram incluídos 408 estudantes. Na graduação, mulheres relataram sentirem-se mais ansiosas ao realizar tratamento em pacientes com suspeita de COVID-19 (54\%) e sentem mais medo ao ouvir que a infecção tem causado mortes (92,4\%), na pós-graduação, responderam ser mais nervosas para conversar com pacientes em ambientes fechados em comparações com homens $(\mathrm{P}<0,05)$. Alunos em fase pré-clínica possuem significativamente menor receio $(65,5 \%)$, ansiedade $(32,3 \%)$ e nervosismo $(28,3 \%)$ do contágio do COVID-19 quando comparados com aqueles na fase clínica. Conclusões: Mulheres e alunos na fase clínica apresentam maior ansiedade e nervosismo.

Descritores: Ansiedade; Estudantes de Odontologia; Medo; Infecções por Coronavírus.

\section{Abstract}

Objectives: This study aimed to investigate the perceptions of dental students regarding fear and anxiety in relation to the management of patients and the risk of infection in the context of the COVID-19 pandemic. Material and methods: This cross-sectional study involved all students regularly enrolled in the School of Dentistry at the Federal University of Pelotas in the first semester of 2020. A questionnaire was applied, collecting demographic data, level of education and questions regarding perceived fear and anxiety in relation to the COVID-19 pandemic. Comparisons were performed according to the phase within the undergraduation (pre-clinical or clinical phase), undergraduation and graduation level, and sex. Independent analyses were performed for undergraduation and graduation students regarding sex $(\alpha<5 \%)$. Results: It was included 408 dental students. Among undergraduation, women reported feeling more anxious when carrying out treatment in patients with suspected COVID-19 (54\%) and feeling more afraid when they heard that infection can lead to death (92.4\%). In the graduate level, women reported to be more nervous when talking to patients in closed vicinity compared to men $(\mathrm{P}<0.05)$. Students in the pre-clinical phase feel significantly less fear (65.5\%), anxiety (32.3\%) and nervousness (28.3\%) regarding COVID-19 when compared with those in the clinical phase. Conclusion: Women and students at more advanced levels of training have increased levels of anxiety and nervousness.

Descriptors: Anxiety; Students, Dental; Fear; Coronavirus Infections.

\section{Resumen}

Objetivo: Este estudio investigó las percepciones del miedo y la ansiedad de los estudiantes de odontología en relación con el manejo de pacientes y el riesgo de infección por COVID-19. Materiales y métodos: Este estudio transversal involucró todos los estudiantes regularmente matriculados en Odontología, en el primer semestre de 2020, en la Universidad Federal de Pelotas. Se aplicó un cuestionario, recogiendo datos demográficos, nivel educativo y preguntas relacionadas con el miedo y la ansiedad ante la pandemia COVID-19. Se realizaron cuatro comparaciones según la fase de pregrado (fase preclínica o clínica), nivel de pregrado y posgrado y según sexos. Se realizaron análisis independientes para comparaciones entre sexos para estudiantes de pregrado y posgrado $(\alpha<5 \%)$. Resultados: Se incluyeron 408 estudiantes. En estudiantes de pregrado, las mujeres informaron sentirse más ansiosas cuando se sometían al tratamiento en pacientes con sospecha de COVID-19 (54\%), ellas sienten más miedo al escuchar que la infección ha causado muertes (92,4\%), en el posgrado, ellas respondieron que estaban más nerviosos al hablar con los pacientes en ambientes cerrados en comparación con los hombres ( $\mathrm{P}<0.05)$. Los estudiantes en la fase preclínica tienen significativamente menos miedo $(65,5 \%)$, ansiedad $(32,3 \%)$ y nerviosismo $(28,3 \%)$ al contagio de COVID-19 en comparación con los de la fase clínica. Conclusión: Las mujeres y los estudiantes en la fase clínica tienen mayor ansiedad y nerviosismo.

Descriptores: Ansiedad; Estudiantes de Odontología; Miedo; Infecciones por Coronavirus.

INTRODUÇÃO

As pandemias experimentadas pela humanidade ao longo dos anos, além de causarem grandes perdas populacionais, abalam famílias emocionalmente, financeiramente e psicologicamente $^{1}$. A mais recente pandemia enfrentada é a "Coronavirus Disease-2019" (COVID-19), que foi decretada como uma pandemia de saúde pública, em 11 de março de 2020, pela Organização Mundial de Saúde (OMS) ${ }^{2}$. Devido à infectividade e alta taxa de contágio do vírus ${ }^{3}$, medidas preventivas foram e têm sido realizadas ao redor do mundo, visando diminuir o número de novas infecções. Dentro dessas medidas, destaca-se o distanciamento social, que levou empresas a optarem pelo home-office, eventos e congressos a serem cancelados e a suspensão de atividades presenciais em escolas e universidades ${ }^{4}$.

É válido destacar que os profissionais de saúde estão sujeitos a um maior risco de contágio por essa doença, destacando-se os profissionais da Odontologia devido aos procedimentos geradores de aerossóis ${ }^{5}$. Pela alta transmissibilidade da doença, o risco de infecção cruzada entre paciente e dentista torna-se alarmante ${ }^{6}$, ocasionando nesses profissionais preocupações com as adequações estruturais e de biossegurança necessárias para prosseguir com a realização de seus atendimentos neste período ${ }^{7,8}$. Além disso, há o medo de um possível contágio, que, 
uma vez ocorrido, pode acarretar na transmissão da enfermidade para familiares, professores, amigos e até outros pacientes ${ }^{9}$.

Para as faculdades de odontologia, a pandemia de COVID-19 apresenta-se como um desafio, pois é necessário pensar na saúde dos alunos, professores, pacientes e funcionários, além de continuar, dentro do possível, o fornecimento de tratamento a população e garantir o ensino odontológico de qualidade ${ }^{10}$. $\mathrm{O}$ estudante de odontologia durante a sua formação pode apresentar níveis de estresse mais elevado que a população em geral $^{11}$, a revisão sistemática realizada por Elani et al. ${ }^{12}$ confirma esse dado e atribui como principal fonte de estresse a alta exigência da necessidade de treinamento odontológico. No contexto da pandemia que enfrentamos, os estudantes ainda precisam lidar com o processo ensinoaprendizagem prejudicado devido à suspensão ou restrição das atividades presenciais. Além disso, os longos períodos em casa, a ausência de atividades estudantis, o atraso na conclusão do curso, a preocupação com o impacto da recessão no mercado de trabalho e o receio da contaminação própria ou de familiares e amigos pelo vírus, ocasionando sentimentos de incerteza, ansiedade e estresse nos estudantes universitários ${ }^{13}$. Dessa forma, o presente estudo teve como objetivo investigar as percepções de estudantes de graduação e pós-graduação em Odontologia de uma universidade pública do sul do Brasil quanto ao medo e à ansiedade em relação ao manejo de pacientes e o risco de infecção no contexto da pandemia de COVID-19.

\section{MATERIAL E MÉTODO}

\section{- Amostra e considerações éticas}

Este estudo observacional transversal foi realizado com alunos da graduação e pós-graduação da Faculdade de Odontologia (FO) da Universidade Federal de Pelotas (UFPel). O estudo foi aprovado pelo comitê de ética da FO-UFPel (parecer número 4.087.139), e os participantes leram o termo de consentimento livre e esclarecido, manifestando o interesse em participarem do presente estudo e permitindo o uso dos dados do questionário. No primeiro semestre de 2020, a faculdade contava com 474 alunos de graduação e 105 alunos de pósgraduação sendo que todos os alunos regularmente matriculados no período da coleta foram convidados a participar. A coleta de dados ocorreu no período de junho a agosto de 2020 .

\section{- Questionário}

Os participantes responderam a um questionário eletrônico, desenvolvido na ferramenta Google Forms que foi divulgado por meio de contatos via e-mail, redes sociais, contato com representantes de turma e divulgação em projetos da instituição. $\mathrm{O}$ questionário constituiu-se em 08 perguntas sobre dados sociodemográficos, nível de formação a ser cursado no primeiro semestre de 2020 e perguntas relacionadas ao medo e ansiedade em relação à pandemia de COVID- 19 (Tabela 1), sendo a maioria das perguntas em formato de múltipla escolha. Essas perguntas foram traduzidas e adaptadas do questionário de Ahmed et al. ${ }^{14}$. Por esse estudo ter envolvido apenas estudantes de odontologia e com o intuito de padronizar a coleta de dados, três questões do questionário foram removidas. Elas são "Do You want to Close Your Dental Practice until the Number of COVID-19 Cases Starts Declining?", “Are you afraid of getting quarantined if get infected?" e "Are You Anxious about the Cost of Treatment if You Get Infected?".

As respostas foram coletas automaticamente pela ferramenta, gerando uma tabela de dados no Microsoft Excel. O banco de dados foi codificado e tabulado para realizar as análises estatísticas.

\section{- Análise estatística}

Para cada questão coletada, quatro comparações distintas foram realizadas, de acordo com o nível de formação (graduação e pósgraduação), nível de formação dentro da graduação (fase pré-clínica ou clínica) e de acordo com os sexos (masculino e feminino). Análises independentes, para as comparações entre os sexos, foram realizadas para os alunos de graduação e de pós-graduação. A fase pré-clínica foi definida com aqueles alunos matriculados entre o $1^{\circ}$ e $2^{\circ}$ anos da graduação. Para as respostas relacionadas a pandemia de COVID-19 a amostra foi dicotomizada em "sim", para o grupo de estudantes que respondeu "sim", ou "não" para os participantes que responderam "não" ou "não sabe". Análises descritivas foram efetuadas e comparações foram realizadas por meio do teste de qui-quadrado ou exato de Fisher. Para todas as comparações, adotou-se um valor de $\mathrm{p}<0,05$ para a significância estatística. Os dados foram analisados pelo software SPSS 25.0 para Windowns (IBM corp., SPSS, Inc., Chicago, IL, USA).

\section{RESULTADOS}

Dos 579 alunos da escola, nove foram excluídos por não estarem regularmente matriculados durante o período da coleta de dados, permanecendo 570 alunos potencialmente elegíveis. Desses, 408 estudantes responderam à pesquisa, sendo 331 da graduação (taxa de resposta: 71,18\%) e 77 da pósgraduação (taxa de resposta: 74,29\%). Ao se analisar as taxas de não resposta, verificou-se que 51 homens e 91 mulheres da graduação não participaram e 10 homens e 17 mulheres que cursam pós-graduação não responderam à pesquisa. Diferenças significativas, entre os sexos, para ambos os níveis de formação, graduação e pós-graduação, não foram observadas ao se comparar os respondentes e não respondentes $(\mathrm{P}>0,05)$. 
Tabela 1. Questionário medo e ansiedade frente à pandemia de Covid-19

\begin{tabular}{|c|c|}
\hline Categoria e Pergunta & $\begin{array}{l}\text { Opções de } \\
\text { resposta }\end{array}$ \\
\hline \multicolumn{2}{|l|}{ Dados Sociodemográficos } \\
\hline \multirow{3}{*}{ Qual seu gênero? } & Feminino \\
\hline & Masculino \\
\hline & Outro \\
\hline \multicolumn{2}{|l|}{ Nível de formação } \\
\hline $\begin{array}{l}\text { Alunos de graduação - qual semestre você está cursando em } \\
2020 / 1^{*}\end{array}$ & Pergunta aberta \\
\hline \multirow{2}{*}{ Alunos da pós-graduação - você está matriculado em que nível? } & Mestrado \\
\hline & Doutorado \\
\hline \multicolumn{2}{|l|}{ Relacionadas à pandemia de COVID- 19} \\
\hline \multirow{3}{*}{$\begin{array}{l}\text { "Você sente medo quando ouve que as pessoas estão } \\
\text { morrendo por causa do COVID-19?" }\end{array}$} & Sim \\
\hline & Não \\
\hline & Não sabe \\
\hline \multirow{3}{*}{$\begin{array}{l}\text { "Você tem medo de levar a infecção da sua clínica } \\
\text { odontológica para a sua família?" }\end{array}$} & Sim \\
\hline & Não \\
\hline & Não sabe \\
\hline \multirow{3}{*}{$\begin{array}{l}\text { "Você se sente nervoso ao conversar com pacientes em } \\
\text { ambientes fechados?" }\end{array}$} & Sim \\
\hline & Não \\
\hline & Não sabe \\
\hline \multirow{3}{*}{$\begin{array}{l}\text { "Você se sente ansioso em fornecer tratamento a um paciente } \\
\text { que está tossindo ou com suspeita de estar Infectado com } \\
\text { COVID-19?" }\end{array}$} & Sim \\
\hline & Não \\
\hline & Não sabe \\
\hline \multirow{3}{*}{$\begin{array}{l}\text { "Você está com medo de se infectar com COVID-19 de um } \\
\text { paciente ou colega de trabalho?" }\end{array}$} & Sim \\
\hline & Não \\
\hline & Não sabe \\
\hline
\end{tabular}

Quanto às perguntas direcionadas para a pandemia de COVID-19 ao se comparar alunos da graduação e da pós-graduação (Figura 1) somente a pergunta "Você se sente ansioso em fornecer tratamento a um paciente que está tossindo ou com suspeita de estar Infectado com COVID-19?" mostrou-se estatisticamente significante, sendo que $49,2 \%$ dos alunos de graduação e $67,9 \%$ dos alunos da pós-graduação responderam "sim" para a questão, revelando uma maior ansiedade entre os pósgraduandos $(\mathrm{p}=0,002)$.

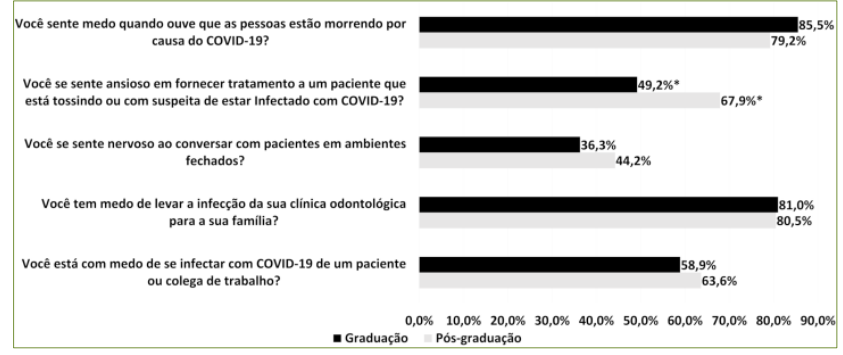

Figura 1: Comparação quanto ao nível de formação cursado no primeiro semestre de 2020

Legenda: $* P<0,05$.

Ao comparar os sexos entre a graduação (Figura 2), às perguntas "Você se sente ansioso em fornecer tratamento a um paciente que está tossindo ou com suspeita de estar Infectado com COVID-19?" e "Você sente medo quando ouve que as pessoas estão morrendo por causa do COVID-19?" mostraram uma diferença significativa entre os grupos. Na primeira pergunta $54 \%$ das mulheres e $39,3 \%$ dos homens responderam "sim", indicando uma maior ansiedade feminina no atendimento de pacientes suspeitos $(\mathrm{P}=0,012)$. Para a segunda pergunta, $92,4 \%$ das mulheres e $71,0 \%$ dos homens responderam "sim" para sentir medo, revelando o maior medo do gênero feminino frente à letalidade da doença $(\mathrm{P}<0,001)$.

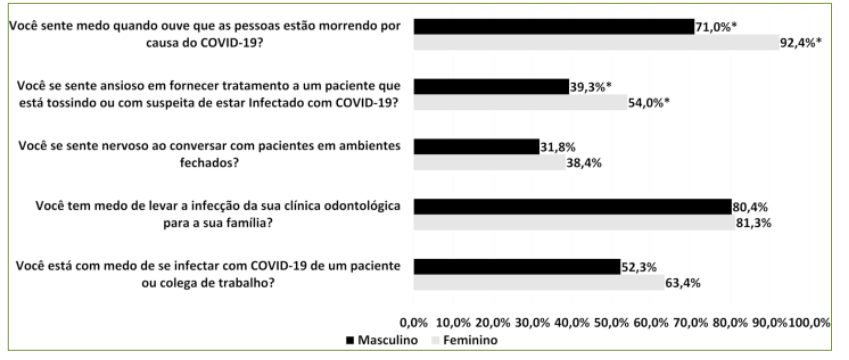

Figura 2: Comparação quanto a variável sexo para os alunos de graduação do primeiro semestre de 2020 . Legenda: $* P<0,05$

Para as comparações entre os alunos de pósgraduação (Figura 3), foi encontrado diferenças significativas apenas para a pergunta: "Você se sente nervoso ao conversar com pacientes em ambientes fechados?", sendo que $57,1 \%$ das mulheres e $9,5 \%$ dos homens responderam "sim", revelando, mais uma vez, o maior nervosismo feminino $(\mathrm{P}<0,001)$. Ao se dividir os graduandos em fase pré-clínica $\left(1^{\circ}\right.$ e $2^{\circ}$ anos) e clínica ( $3^{\circ}, 4^{\circ}$ e $5^{\circ}$ anos), $32,3 \%$ dos alunos que estão na pré-clínica e $59,8 \%$ dos alunos da clínica responderam "sim" para a questão "Você se sente ansioso em fornecer tratamento a um paciente que está tossindo ou com suspeita de estar Infectado com COVID-19?", mostrando a maior preocupação dos alunos que realizam algum tipo de atendimento ao público $(\mathrm{P}<0,001)$. Para a pergunta "Você se sente nervoso ao conversar com pacientes em ambientes fechados?", 41,2\% dos estudantes em fase clínica e $28,3 \%$ dos em fase pré-clínica responderam "sim", o que evidência a maior tranquilidade dos alunos préclínicos ao falarem com os pacientes $(\mathrm{P}=0,018)$. Quanto à pergunta "Você tem medo de levar a infecção da sua clínica odontológica para a sua família?" $88,7 \%$ dos anos mais avançados e $68,5 \%$ dos anos iniciais responderam "sim", esse resultado foi estatisticamente significante $(\mathrm{P}<0,001)$ (Figura 4).

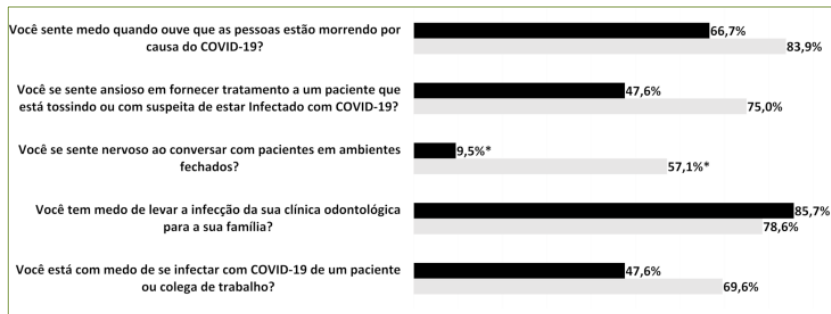

$0,0 \% \quad 10,0 \% \quad 20,0 \% \quad 30,0 \% \quad 40,0 \% \quad 50,0 \% 60,0 \% \quad 70,0 \% \quad 80,0 \% 90,0 \%$

Figura 3: Comparação quanto a variável sexo para os alunos da pós-graduação do primeiro semestre de 2020. Legenda: $* P<0,05$.

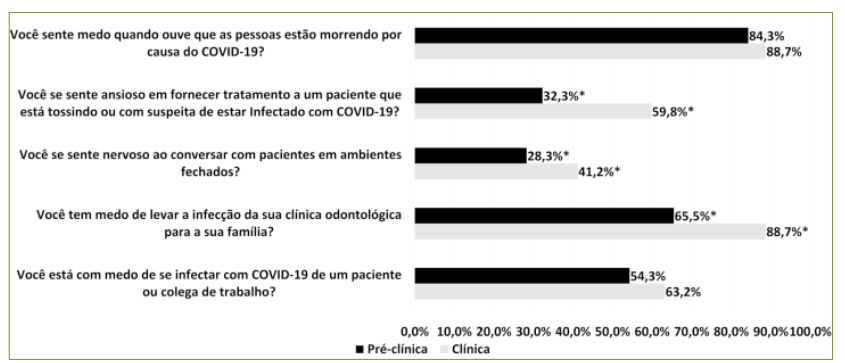

Figura 4. Comparação quanto ao nível de formação na graduação cursado no primeiro semestre de 2020. Legenda: $* P<0,05$ 


\section{DISCUSSÃO}

O rápido avanço do número de infecções por COVID-19, as repentinas orientações sobre isolamento social, o crescente número de mortes e o impacto econômico causado pela pandemia, inevitavelmente, afetam o bem estar psicológicos da população, sendo o impacto muitas vezes agravado em pacientes do grupo de risco, idosos, crianças e profissionais da saúde ${ }^{15}$. Para o cirurgião-dentista, profissional da saúde que tem seu trabalho diário amplamente afetado pela doença, o medo de ser infectado com COVID-19, o risco da contaminação de algum membro da família e a necessidade de informações suficientes sobre o vírus vêm sendo associados a maior sobrecarga e sofrimento psicológico da categoria ${ }^{16}$.

O presente estudo buscou fornecer um panorama geral sobre os impactos da pandemia de COVID-19, na saúde mental de estudantes de odontologia, permitindo uma previsão e avaliação sobre os sentimentos dos alunos diante de um possível retorno das atividades presenciais. $\mathrm{O}$ estudo revelou que aproximadamente metade dos alunos mostrou-se ansioso com a possibilidade de contrair o vírus de um colega de trabalho ou paciente, sendo esse número visivelmente maior quando questionado sobre a possível transmissão para familiares. Esse resultado corrobora com o estudo de Rymarowicz et al. $^{17}$, em que $80,7 \%$ dos cirurgiões-dentistas apresentavam medo de contrair a doença de um paciente ou colega de trabalho e 90,1\% temiam transmitir o vírus para familiares. Essa preocupação pode ser justificada não só devido a presença de grupos com maior risco de infecção, como também ao fato da possível contaminação por outras doenças já ser aceita pelos profissionais como parte do risco ocupacional. Porém, devido ao pouco conhecimento dos sintomas, incertezas sobre métodos preventivos e rápida propagação, o medo da transmissão para seus familiares torna-se elevado ${ }^{18}$.

Uma diferença significativa entre os gêneros foi apresentada, tendo as mulheres maior ansiedade no manejo do paciente e medo frente à letalidade da pandemia. Depressão e ansiedade são sintomas mais comuns entres as mulheres dos que entre os homens $^{19,20}$, podendo a prevalência de sintomas de ansiedade durante a pandemia de COVID- 19 ser três vezes maior do que em homens ${ }^{21}$. A maior facilidade feminina para articulação das suas emoções e o maior número de mulheres ocupando cargos nas profissões mais afetadas pela pandemia ${ }^{22}$, além da maior propensão feminina para o desenvolvimento de estresse pós-traumático em situações pandêmicas ${ }^{23}$ são algumas justificativas para os achados.

Para as comparações entre os níveis de formação durante a graduação, os alunos pré-clínicos mostraram-se menos preocupados e ansiosos em relação aos alunos em fase clínica. Esses resultados podem ser justificados por outros estudos, realizados em períodos acadêmicos normais (anterior à pandemia), como no estudo transversal de TERÁN que encontrou que alunos na fase clínica apresentam risco 2,56 vezes maior de ter estresse que os alunos em fases pré-clínica ${ }^{24}$. Esse maior desconforto dos alunos, em anos mais avançados, pode ser justificado pela maior carga horária de aulas práticas, responsabilidade com os pacientes, negligência com a vida pessoal, preocupações com o mercado de trabalho, medo de reprovação e preocupação com a saúde $^{25}$. O aprendizado somente em laboratório dos semestres iniciais pode justificar a menor preocupação com o manejo dos pacientes por parte destes, o que também foi encontrado no estudo de Agius et al. ${ }^{26}$, que revelou que os alunos sem contato direto com pacientes possuíam menor medo da contaminação pelo vírus da COVID-19.

Apesar da ausência das atividades presenciais e dos sentimentos de medo e ansiedade proporcionados pela pandemia é necessário que as instituições estabeleçam vínculo com os alunos com o objetivo de mantê-los motivados e engajados com o aprendizado da odontologia. Ferramentas de elearning, como salas virtuais e questionários, podem cumprir esse objetivo além de permitirem informar os alunos quanto as particularidades do atendimento odontológico frente ao COVID-19, instigando-os também, a tornar esse conhecimento mais acessível a população ${ }^{27}$. Serviços de saúde mental no formato remoto (online) vem sendo amplamente implementados, permitindo um acompanhamento psicológico de fácil acesso para aqueles indivíduos que apresentam sintomas psicológicos derivados da pandemia $^{28}$.

É importante destacar algumas limitações do presente estudo. O caráter transversal, não permitindo temporalidade deve ser considerado. Além disso, sabe-se que as diferentes curvas de transmissibilidade do COVID-19 são mutáveis em um curto intervalo de tempo. Essas mudanças podem ter impacto significativo nas percepções de medo e ansiedade ${ }^{29}$. Entretanto, o presente estudo envolveu estudantes de odontologia de apenas uma instituição, em um período de coleta relativamente curto, o que pode ajudar a diminuir essa variabilidade.

\section{CONCLUSÃO}

Com base nos resultados, concluiu-se que as mulheres apresentam maior ansiedade em atender pacientes com suspeita de infecção, são mais nervosas para conversar com pacientes em ambientes fechados e sentem mais medo ao ouvir que a infecção com COVID-19 tem causado mortes. Concluiu-se também que alunos em semestres iniciais, sem contato com as atividades clínicas, possuem menos receio, ansiedade e nervosismo do contágio do COVID-19 quando comparado com aqueles que já 
atendem pacientes. Além disso, efeitos negativos na saúde mental dos estudantes devido a um período de vida atípico e desafiador ocasionado pela pandemia de COVID-19 necessita ser investigado, assim como a implementação de estratégias de promoção de saúde mental necessitam ser realizadas.

\section{AGRADECIMENTOS}

O presente estudo foi conduzido em um programa de Pós-graduação financiado pela Coordenação de Aperfeiçoamento de Pessoal de Nível Superior - Brasil (CAPES) - Código 001.

\section{REFERÊNCIAS}

1. Chang J, Yuan Y, Wang D. [Mental health status and its influencing factors among college students during the epidemic of COVID-19]. Nan Fang Yi Ke Da Xue Xue Bao. 2020;40(2):171-176.

2. WHO. World Health Organization. WHO Director-General's opening remarks at the media briefing on COVID-19- 11 March 2020. 2020. Disponível em:

https://www.who.int/dg/speeches/detail/whodirector-general-s-opening-remarks-at-the-mediabriefing-on-covid-19---11-march-2020. Acesso em: 8 de novembro de 2020 .

3. Pascarella G, Strumia A, Piliego C, Bruno F, Del Buono R, Costa F, Scarlata S, Agrò FE. COVID19 diagnosis and management: a comprehensive review. J Intern Med. 2020;288(2):192-206.

4. Chen E, Lerman K, Ferrara E. Tracking Social Media Discourse About the COVID-19 Pandemic: Development of a Public Coronavirus Twitter Data Set. JMIR Public Health Surveill. 2020;6(2):e19273.

5. Iyer P, Aziz K, Ojcius DM. Impact of COVID-19 on dental education in the United States. J Dent Educ. 2020;84(6):718-722.

6. Meng L, Hua F, Bian Z. Coronavirus Disease 2019 (COVID-19): Emerging and Future Challenges for Dental and Oral Medicine. J Dent Res. 2020;99(5):481-487.

7. Peng X, Xu X, Li Y, Cheng L, Zhou X, Ren B. Transmission routes of 2019-nCoV and controls in dental practice. Int J Oral Sci. 2020;12(1):9.

8. Machado RA, Bonan PRF, Perez DEDC, Martelli Júnior H. COVID-19 pandemic and the impact on dental education: discussing current and future perspectives. Braz Oral Res. 2020;34:e083.

9. Ataş $O$, Talo Yildirim T. Evaluation of knowledge, attitudes, and clinical education of dental students about COVID-19 pandemic. PeerJ. 2020;8:e9575.

10. Deery C. The COVID-19 pandemic: implications for dental education. Evid Based Dent. 2020;21(2):46-47.

11. Basudan S, Binanzan N, Alhassan A. Depression, anxiety and stress in dental students. Int J Med Educ. 2017;8:179-186.
12. Elani HW, Allison PJ, Kumar RA, Mancini L, Lambrou A, Bedos C. A systematic review of stress in dental students. J Dent Educ. 2014; 78(2):226-42.

13. Sahu P. Closure of Universities Due to Coronavirus Disease 2019 (COVID-19): Impact on Education and Mental Health of Students and Academic Staff. Cureus. 2020;12(4):e7541.

14. Ahmed MA, Jouhar R, Ahmed N, Adnan S, Aftab M, Zafar MS, Khurshid Z. Fear and Practice Modifications among Dentists to Combat Novel Coronavirus Disease (COVID-19) Outbreak. Int J Environ Res Public Health. 2020;17(8):2821.

15. Talevi D, Socci V, Carai M, Carnaghi G, Faleri S, Trebbi E, di Bernardo A, Capelli F, Pacitti F. Mental health outcomes of the CoViD-19 pandemic. Riv Psichiatr. 2020;55(3):137-44.

16. Mijiritsky E, Hamama-Raz Y, Liu F, Datarkar AN, Mangani L, Caplan J, Shacham A, Kolerman R, Mijiritsky O, Ben-Ezra M, Shacham M. Subjective Overload and Psychological Distress among Dentists during COVID-19. Int J Environ Res Public Health. 2020;17:5074.

17. Rymarowicz J, Stefura T, Major P, Szeliga J, Wallner G, Nowakowski M, Pędziwiatr M. General surgeons' attitudes towards COVID-19: A national survey during the SARS-CoV-2 virus outbreak. Eur Surg. 2020;1-6.

18. Adams JG, Walls RM. Supporting the Health Care Workforce During the COVID-19 Global Epidemic. JAMA. 2020;323(15):1439-40.

19. Naz N, Iqbal S, Mahmood A. Stress, anxiety and depression among the dental students of university college of medicine and dentistry Lahore; Pakistan. Pak J Med Health Sci. 2017;11(4):1277-81.

20. Waqas A, Iftikhar A, Malik Z, Aedma KK, Meraj $\mathrm{H}$, Naveed S. Association of severity of depressive symptoms with sleep quality, social support and stress among Pakistani medical and dental students: A cross-sectional study. Global Psychiatry. 2019;2(2):211-20.

21. Wang Y, Di Y, Ye J, Wei W. Study on the public psychological states and its related factors during the outbreak of coronavirus disease 2019 (COVID-19) in some regions of China. Psychol Health Med. 2020;1-10.

22. Xiong J, Lipsitz O, Nasri F, Lui LMW, Gill H, Phan L, Chen-Li D, Iacobucci M, Ho R, Majeed A, McIntyre RS. Impact of COVID-19 pandemic on mental health in the general population: A systematic review. J Affect Disord. 2020;277:55-64.

23. Liu N, Zhang F, Wei C, Jia Y, Shang Z, Sun L, Wu L, Sun Z, Zhou Y, Wang Y, Liu W. Prevalence and predictors of PTSS during COVID-19 outbreak in China hardest-hit areas: Gender differences matter. Psychiatry Res. 2020;287;112921.

24. Terán E, Mayta-Tovalino F. Risk Factors, Self- 
perceived Stress, and Clinical Training among Dentistry Students in Peru: A Cross-sectional Study. J Contemp Dent Pract. 2019;20(5):561-5.

25. Uraz A, Tocak YS, Yozgatligil C, Cetiner S, Bal B. Psychological well-being, health, and stress sources in Turkish dental students. J Dent Educ. 2013:77(10):1345-55.

26. Agius AM, Gatt G, Vento Zahra E, Busuttil A, Gainza-Cirauqui ML, Cortes ARG et al. Selfreported dental student stressors and experiences during the COVID-19 pandemic. J Dent Educ. 2020. doi: $10.1002 /$ jdd. 12409 .

27. Hu J, Zou H, Dai Y, Feng Z. How to keep students engaged in oral health education during the COVID-19 pandemic. J Dent Educ. 2020. doi: 10.1002/jdd.12420.

28. Liu S, Yang L, Zhang C, Xiang YT, Liu Z, Hu S, Zhang B. Online mental health services in China during the COVID-19 outbreak. Lancet Psychiatry. 2020;7(4):e17-8.

29. Maia BR, Dias PC. Anxiety, depression and stress in university students: the impact of COVID-19. Estudos de Psicologia (Campinas). 2020;37:e200067.

\section{CONFLITO DE INTERESSES}

Os autores declaram não haver conflitos de interesse.

\section{AUTOR PARA CORRESPONDÊNCIA}

Francisco Wilker Mustafa Gomes Muniz

Universidade Federal de Pelotas - UFPEL

Rua Gonçalves Chaves, 457

960105-560 Pelotas - RS, Brasil

Telefone: +55 (53) 991253611

E-mail: wilkermustafa@gmail.com
Submetido em 20/11/2020

Aceito em 14/12/2020 